Particle physics

\title{
The F meson found at Cornell
}

\section{from J. M. Yelton}

PARTICLE physicists using the CLEO detector at Cornell ${ }^{1}$ have recently shown the most compelling evidence yet of the existence of the $\mathrm{F}^{+}$meson, the combination of a charm quark with a strange anti-quark ${ }^{2}$. They measure the invariant mass of the $\mathrm{F}^{+}$to be $1970 \mathrm{MeV} / \mathrm{c}^{2}$, filling an important gap in physicists knowledge of charmed mesons.

The discovery of the $\mathrm{J} / \psi$ in 1974 started a new era of particle physics and its identification as a bound state of a charm quark with a charm anti-quark was seen as a validation of the quark model of hadronic matter. The model then predicted a whole spectrum of mesons containing charm quarks. Combination of charm quarks with up and down anti-quarks, the $\mathrm{D}^{0}$ and $\mathrm{D}^{+}$, were found in 1975, and their excited states, the $\mathrm{D}^{* 0}$ and $\mathrm{D}^{*}+$ followed soon after. However, the $\mathrm{F}^{+}$meson, the combination of a charm quark with a strange anti-quark, has proved difficult to detect. The properties of $\mathrm{F}^{+}$mesons may be predicted by analogy with $D$ mesons. The $F$ mass is expected to be somewhat greater than that of the $\mathrm{D}^{+}\left(1,868 \mathrm{MeV} / \mathrm{c}^{2}\right)$, and its decay products to be typically an $\eta$ or a $\phi$ meson together with a number of pions. The CLEO collaboration has sought for, and found, a resonance that decays into $\phi \pi^{+}$and which they identify with the $\mathrm{F}^{+}$meson.

The CESR $\mathrm{e}^{+} \mathrm{e}^{-}$collider at Cornell produces collision energies of around 10 $\mathrm{GeV}$, just sufficient to create mesons including bottom quarks, but also fairly well matched for studies of charmed mesons. The CLEO detector is a general purpose detector operating at CESR, and incorporates good charged particle momentum measurement. The first stage in their analysis is to make the invariant mass combination of oppositely charged tracks under the hypothesis that they are kaons (tracks clearly identified as pions are excluded). A clear signal corresponding to the $\phi$ meson (mass $=1,020 \mathrm{MeV} / c^{2}$ ) is seen. Those combinations with mass within $5 \mathrm{MeV} / \mathrm{c}^{2}$ of the $\phi$ mass were taken as $\phi$ candidates. Combinations were then made of the $\phi$ candidates with each remaining pion in the event. The invariant mass spectrum for $\phi \pi^{+}$combinations with momentum greater than $2.5 \mathrm{GeV} / c^{2}$ is shown in the figure. A clear peak is seen, with a width consistent with that due to experimental resolution alone and an invariant mass measured to be $1970 \pm 5 \pm 5$ $\mathrm{MeV} / \mathrm{c}^{2}$, where the errors are statistical and systematic respectively. The probability that the peak is due to a statistical fluctuation alone is around 1 in 20,000, and its decay characteristics are consistent with it being an $\mathrm{F}^{+}$. Although confirmation of the signal in another channel (for example, $\mathrm{F}^{+} \rightarrow \phi \pi^{+} \pi^{-} \pi^{+}$would be very welcome, there seems little doubt that the resonance observed by CLEO is, indeed, an $\mathrm{F}^{+}$ meson.

Previous experiments have shown some evidence for the existence of $\mathrm{F}^{+}$, produced

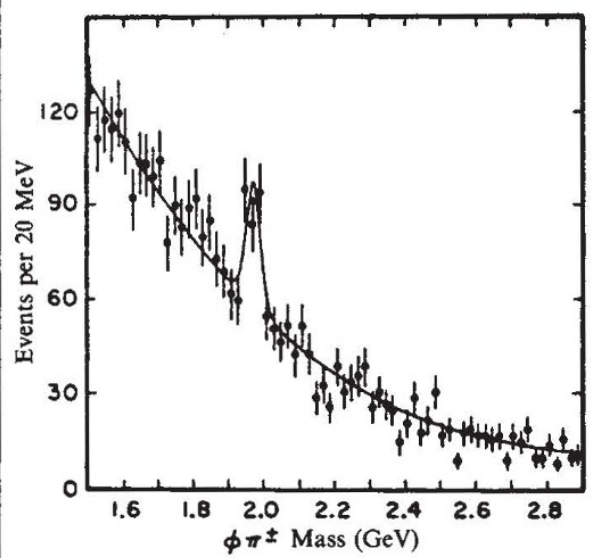

by a variety of mechanisms and decaying into an $\eta$ and pions ${ }^{3,4}$, and also into $a \phi$ and $\mathrm{a}^{+}$(ref. 5). Although the mass measurement from each individual experiment was weak they tended to indicate an $\mathrm{F}^{+}$mass of around $2,030 \mathrm{MeV} / \mathrm{c}^{2}$, so the CLEO result has come as something of a surprise. Theoretically, the exact value of the $\mathrm{F}^{+}$ mass is interesting as it helps constrain models of the shape of the potential force binding together the quark and anti-quark. In principle, from a knowledge of this binding force together with a face value for the effective masses of the various flavours of quark, it should be possible to calculate the masses of all mesons. This is made more difficult by the fact that in the charmed mesons the quark masses are small compared with the kinetic energy of the quarks inside the mesons, so relativistic effects are important

The CLEO experiment will not be able to measure the lifetime of the $\mathrm{F}^{+}$produced, but several experiments using optical techniques (bubble chambers and nuclear emulsions) have already tried ${ }^{4}$. Their main problem is unambiguously separating the $\mathrm{F}^{+}$from other charmed partcles. The $\mathrm{F}^{+}$ lifetime is expected to be similar to that of the $\mathrm{D}^{0}$ (around $4 \times 10^{-13} \mathrm{~s}$ ), but there is still some controversy as to the exact decay mechanism of charmed mesons and a precise measurement of the $\mathrm{F}^{+}$lifetimes should help clarify the situation.

At a time when there are big projects being undertaken to create $\mathrm{e}^{+} \mathrm{e}^{-}$collisions of energies around $100 \mathrm{GeV}$, the CLEO result highlights the amount of physics still to be explored at lower mass scales.

J.M. Yelton is at the Stanford Linear Accelerator Centre, Stanford University, P.O. Box 4349, Stanford, Ca 94305.

\footnotetext{
1. A. Chen et al. Phys. Rev. Lett. 51, (1983). The CLEO group is a collaboration from the Universities of Cornell, is a collaboration from the Universities of Cornell, Vanderbilt, and Ithaca College.

2. Reference to a state will always imply the sum of that state and its charge conjugate state.

3. R. Ammar et al. Phys. Lett. 94B, 118 (1979)

4. R. Brandelik et al. Phys. Lett. 80B, 412 (1979). D. Aston et al. Phys, Lett. 100B, 91 (1981).

5. D. Aston et al. Nucl. Phys. B189 205. (1981).
}

\section{Human genetics}

\section{Human gene mapping rolls along}

\section{from Ellen Solomon and Peter Goodfellow}

THE enormous task of mapping the human genome was begun in 1936 using the classical Mendelian techniques of family and population linkage studies. This approach eventually ran into difficulties because there were not enough polymorphic markers available, and in 1970 , it gave way to new methods using somatic cell genetics. The random loss of human chromosomes from human/rodent somatic cell hybrids made it possible to map genes by correlating the presence of human genes, or gene products, with particular human chromosomes. In addition, the large genetic differences between humans and rodents meant that many more genes could be studied. By 1980 the technique had allowed approximately

-Human Gene Mapping 7, to be published by the March of Dimes and Cytogenetics and Cell Genetics. Organised by $\mathbf{R}$. Sparkes, M.A. Spence and T. Mohandas in August, 1983, and held at U.C.L.A.
300 genes to be assigned to particular autosomes - compared with only 20 in the previous 50 years.

Now the new decade has contributed a new revolution: gene cloning. Paradoxically this revolution has completed the circle; the application of DNA probes and associated restriction enzyme site polymorphisms (also known as RFLP for restriction fragment length polymorphisms) can provide an unlimited number of genetic markers for studying human families. Mendelian genetics is once again the major tool for mapping the human genome. Future historians of science will have no difficulty in discerning these trends as they are illustrated by biannual meetings devoted to human gene mapping. The latest* was held in California and was the first fully to reflect the resurgence of the old genetics.

The short-term goal of human gene 